\title{
Long-Term Survival (23 Years) in a 26-Year-old Male after Antineoplaston Therapy for a Progressive, Diffuse Intrinsic Pontine Glioma: A Case Report
}

\author{
Stanislaw R. Burzynski ${ }^{1 *}$, Gregory S. Burzynski ${ }^{1}$, Tomasz Janicki ${ }^{1}$ and Samuel Beenken ${ }^{2}$ \\ ${ }^{1}$ Burzynski Clinic, USA \\ ${ }^{2}$ Calera Alabama, USA
}

*Corresponding author: Stanislaw R Burzynski, MD, PhD, Burzynski Clinic, 9432 Katy Freeway, Houston, TX, 77055, USA, Tel: 713-335-5664, Fax: 713-335-5658

\begin{abstract}
Background: Individuals suffering from diffuse intrinsic pontine glioma (DIPG) face a dismal prognosis with a median overall survival of approximately 11 months, and a 2 -year survival rate of $10 \%$. Long-term survival is very rare. To date, radiotherapy remains the standard of care at diagnosis, but offers a survival benefit of approximately 3 months. Chemotherapy has not shown to be effective. There is no Standard of Care for progressive DIPG after radiation therapy (RT). The purpose of this case report is to present the 23-year survival of a 26-year-old male treated with Antineoplaston therapy A10 and AS2-1 (ANP) for progressive DIPG.
\end{abstract}

Methods: The patient presented here enrolled in BT-3, a Phase II protocol utilizing ANP. Before being enrolled in BT-3, the patient met the following eligibility criteria i) The presence of a measurable high-grade astrocytoma (DIPG); ii) Demonstrated progressive disease (PD) following RT or chemotherapy; iii) A life expectancy of less than three months; and iv) A signed informed consent document indicating that he was aware of the investigational nature of ANP, the unpredictable nature of his response to ANP, and the possibility of worsening disease while receiving ANP. Tumor response was measured by bi-monthly MRIs of the brain. All surviving patients were followed for more than three months.

Results: This patient presented to the Burzynski Clinic (BC) with an anaplastic (high-grade) astrocytoma of the pons (DIPG) with left facial hemiplegia, diplopia, and left hemiparesis with hyperactive left-sided deep tendon reflexes and positive Babinski. The patient was experiencing headaches, drowsiness, visual loss, decreased hearing, dysphagia, and intermittent seizure activity. He was confined to a wheelchair. Following ANP, the patient obtained long term survival ( 23 years). This patient's response reflects the type of outcomes seen in the other 19 patients $(20$ patients total) who were enrolled in BT-3. A complete response (CR) was achieved in six patients $(30 \%)$, a partial response (PR) was achieved in one patient (5\%), stable disease (SD) was achieved in nine patients (45\%), and PD was seen in four patients (20\%).

Conclusion: ANP is an attractive therapeutic option for those patients with progressive high-grade astrocytomas (glial tumors with astrocytic differentiation), including DIPG, who are ineligible for or refuse $\mathrm{RT}$, and/or demonstrate PD following RT or chemotherapy. We present the case of a young male who developed a persistent CR following ANP.

\section{Keywords}

Brain tumor, Anaplastic astrocytoma, Brain stem glioma, Diffuse intrinsic brainstem glioma, Diffuse midline glioma, H3-K27 mutant (DMG H3-K27M), Antineoplaston Therapy, Phase II and III studies
Abbreviations
A-10: Antineoplaston A10 (Atengenal); AE: Adverse Event; ANP: Antineoplaston Therapy (A-10 + AS2-1); AS2-1: Antineoplaston AS2-1 (Astugenal); Astugenal: Antineoplaston AS2-1 (AS2-1); Atengenal: Antineoplaston A10 (A10); BC: Burzynski Clinic; BRI: Burzynski Research Institute; CCNU: Lomustine; CNS: Central Nervous System; CR: Complete Response; CTEP: Cancer Therapy Evaluation Program; CVA: Cerebral Vascular Accident; DIPG: Diffuse Intrinsic Pontine Glioma; DMG H3-K27M: Diffuse Midline Glioma, H3-K27 mutant; FDA: Food and Drug Administration; IV: Intravenous; MRI: Magnetic Resonance Imaging (of the brain); NIH: National Institutes of Health; OR: Objective 
Response; OS: Overall Survival; PD: Progressive Disease; PR: Partial Response; RT: Radiation Therapy; SD: Stable Disease; WHO: World Health Organization; VP: VentriculoPeritoneal

\section{Introduction}

Diffuse intrinsic pontine glioma (DIPG) originates in the glial cells of the pons, an integral part of the brainstem. Gliomas can be low- or high-grade based on histologic criteria and/or MRI findings. Individuals suffering from DIPG face a dismal prognosis with a median overall survival of approximately 11 months, and a 2 year survival rate of $10 \%[1,2]$. Due to the location of the tumor, surgical resection is not possible. To date, radiotherapy (RT) remains the standard of care at diagnosis, but offers a survival benefit of approximately 3 months [3]. Chemotherapy has not shown to be effective [2]. There is no Standard of Care for progressive DIPG after RT.

Brainstem gliomas represent 10 to $20 \%$ of primary tumors of the central nervous system and are diagnosed primarily in children with a median age of onset of 6 to 7 years [4]. The number of children in the USA with brainstem gliomas is approximately 660 [5]. Most brainstem gliomas occur in the pons ("pontine gliomas").

Pontine tumors affect the cranial nerves, causing symptoms such as diplopia (double vision), the inability to fully close the eyelids, drooping of one side of the face, dysphagia (difficulty swallowing), and difficulty chewing. These tumors also affect the "long tracks" of the brain, with resultant weakness of the arms and/or legs.

At the time of diagnosis, these tumors are usually confined to the brainstem. When the disease spreads, the spread is usually contiguous. Metastasis via the subarachnoid space has been reported in up to $30 \%$ of cases and usually occurs at the same time as local disease relapse. There is no generally applied staging system for childhood brainstem gliomas.

Due to its anatomical location, diagnostic biopsies of brainstem gliomas are difficult to obtain. Diagnosis is frequently based on MRI alone. In contrast to the majority of centers in the United States, routine biopsy of children with suspected DIPG has been performed in Europe since 2003 [6]. In a report detailing their experience in 24 children, morbidity was reported in 2 children (cranial nerve palsy, worsening hemiparesis), which was reversible, and no mortality. The investigators concluded that the procedure was relatively safe in experienced hands using modern neurosurgical technique [6]. Given this demonstration of relative safety, there is a movement within the pediatric neurooncology community to push for routine biopsy of patients with suspected DIPG [7].
MRI identifies four different types of brainstem gliomas: focal, dorsal exophytic, cervico-medullary, and DIPG. With the increased use of biopsy for the diagnosis of DIPG, the World Health Organization (WHO), in 2016, reclassified the majority of DIPG within a novel tumor entity: Diffuse midline glioma, H3-K27 mutant (DMG H3-K27M). This entity is defined as an infiltrative highgrade glioma, located in the brain midline, i.e., usually brainstem, spinal cord, cerebellum or thalamus, with astrocytic differentiation and $\mathrm{K} 27 \mathrm{M}$ mutation in either H3F3A or HIST1H3B/C [8]. Up to 85\% of the DIPGs harbor this mutation $[9,10]$. Wild-type H3-K27 DIPGs have not yet been separately classified within the revised WHO classification, but show similar survival as H3-K27M DIPGs [11].

A six-week course of conventional RT is standard therapy for DIPG and frequently will result in improvement of signs and symptoms. Unfortunately, the sign and symptoms usually recur after six to nine months, concomitant with rapid disease progression. In children, survival past 14 to 18 months is uncommon.

Adult DIPG (patient age $>21$ years) is very uncommon with reports of its yearly incidence in the U.S. ranging from 20-100 cases. We have previously reviewed this clinical entity [12]. Whereas pediatric DIPG has a prognosis of 10 months, with only $10 \%$ of pediatric patients living $>2$ years after diagnosis, median survival for adult brainstem glioma is in the range of 3040 months [13].

Growth of normal cells is controlled by cell cycle progression genes (oncogenes) and by cell cycle arrest genes (tumor suppressor genes). In cancer, alteration of these control genes in malignant cells favors aggressive cell proliferation. Evidence suggests that Antineoplaston A-10 (Atengenal, A10) and Antineoplaston AS2-1 (Astugenal, AS2-1) function as "molecular switches" which "turn on" tumor-suppressor genes and "turn off" oncogenes. Hence, the antineoplastic action of ANP involves restoration of cell cycle control, induction of programmed cell death, and interference with cancer cell metabolism and nuclear transport $[14,15]$.

In 1988, following the completion of Phase I studies of A10 and AS2-1, we developed the Phase II protocol designated BT-3 and titled "Therapy of Primary Brain Tumors with Antineoplaston A10 and Antineoplaston AS2-1". The objectives of this protocol were to determine 1) The effectiveness of ANP in the control of primary brain tumors and 2) The toxicity of ANP in patients with primary brain tumors. The 26-year-old male, whose case is detailed herein, was treated under BT-3 for a recurrent DIPG.

\section{Case Description}

The patient had been in good health until the Fall of 1986 when he was evaluated for headaches, blurred 
vision, and incoordination. He was diagnosed via MRI with an anaplastic astrocytoma of the pons (DIPG). Genomic studies, such as mutation of H3-K27 were not performed at the time because such technology was not available. In April 1988, the patient underwent partial resection of the tumor and placement of a ventriculoperitoneal (VP) shunt. In May 1988, he began a course of 7800 cGy hyperfractionated RT. In August 1988, his headaches worsened and were accompanied by marked drowsiness and progressive visual loss. At that time, a second partial resection of the tumor was undertaken and the VP shunt was revised. There was improvement in his symptoms. In August to September 1989, the patient received two courses of lomustine (CCNU) followed by Procarbazine $250 \mathrm{mg}$ daily (10/17/1989 to 10/23/1989).

At the time of his presentation at the Burzynski Clinic (BC), the patient was confined to a wheelchair. He complained of headaches, marked drowsiness, progressive visual loss, decreased hearing, dysphagia,

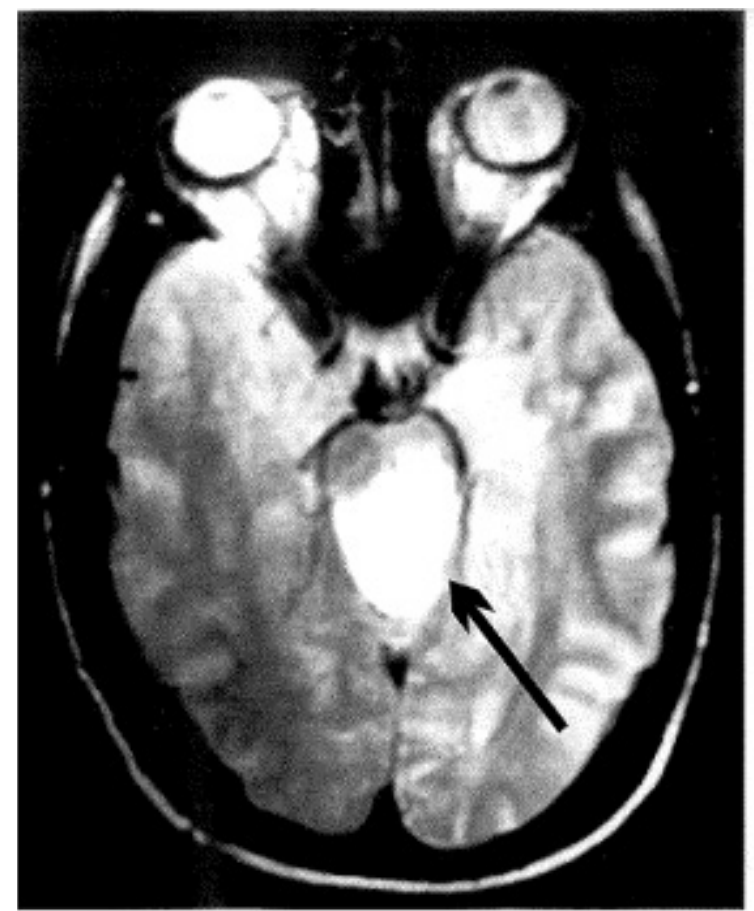

A. Sep. 23,1988

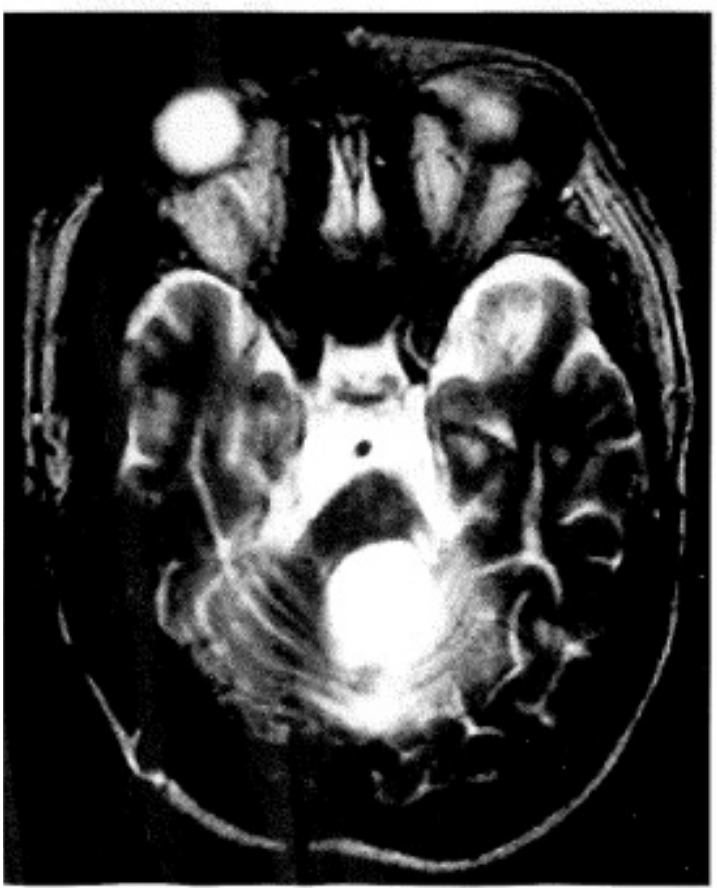

C. Aug. 28, 1995

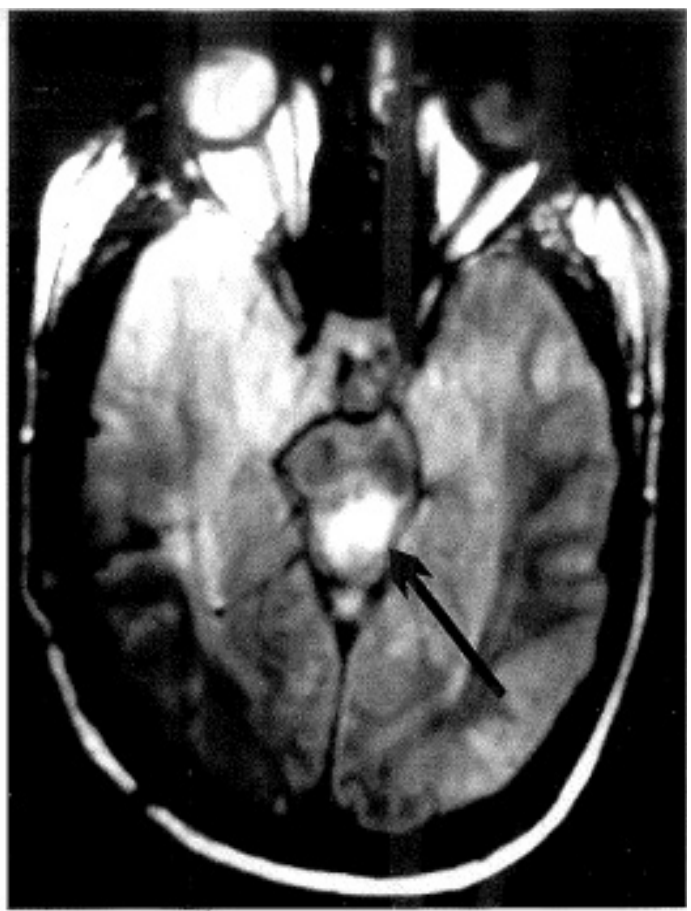

B. Oct. 19,1990

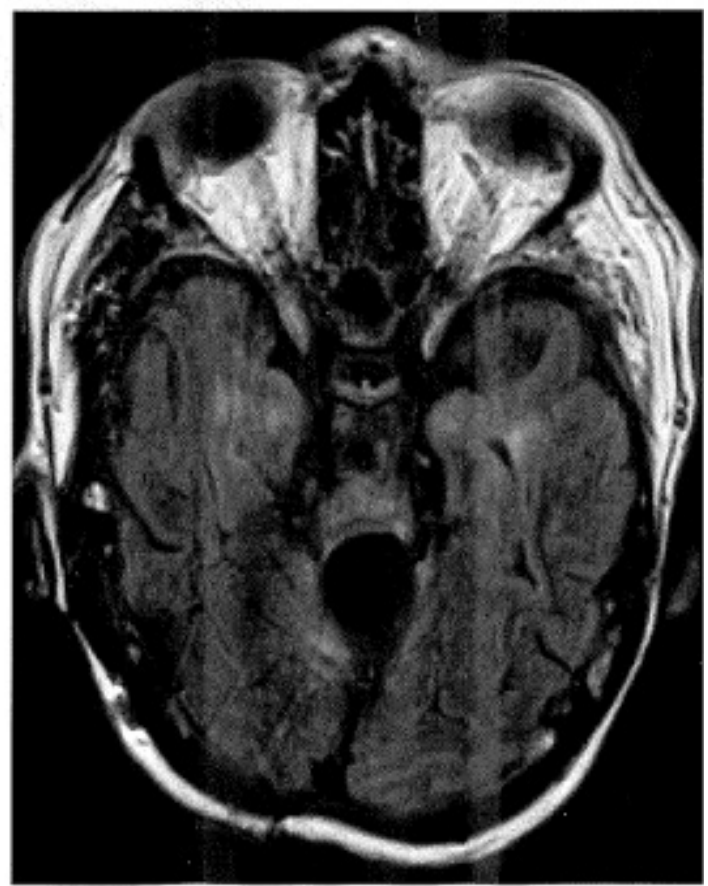

D. Aug. 16, 2012

Figure 1: Axial brain magnetic resonance imaging (MRI) T2 weighted: $1 \mathrm{~A}$. (baseline) demonstrates a high signal intensity mass. $1 \mathrm{~B}$ and $1 \mathrm{C}$ are follow-up images that show a complete response (CR) of the mass to treatment. Figure 1D (FLAIR) also shows the $\mathrm{CR}$. 
and intermittent seizure activity. Neurologic exam revealed left facial hemiplegia, diplopia, left hemiparesis with hyperactive left-sided deep tendon reflexes and a positive Babinski. His KPS was 40. An MRI of the brain, performed on September 23, 1988 (See Figure 1A and Figure 2A), serves as the baseline MRI.

Before being administered ANP in BT-3, the patient meet the following eligibility criteria i) The presence of a measurable high-grade astrocytoma (a glial tumor with astrocytic differentiation); ii) Demonstrated progressive disease (PD) following RT or chemotherapy; iii) A life expectancy of less than three months; iv) A signed informed consent document indicating that he was aware of the investigational nature of ANP, the unpredictable nature of his response to ANP, and the possibility of worsening disease while receiving ANP.

The specifics of therapy and measurement of response under BT-3 have previously been reported [12]. The preferred method of delivery of A10 and AS21 was via a subclavian catheter and infusion pump. A minimum of six months of treatment was necessary for a patient to be evaluable. Patients were removed from treatment secondary to progressive disease, unacceptable toxicity, or patient request.

The patient was treated with ANP delivered by subclavian catheter and infusion pump. A10 was initiated on October 30, 1989 and the dosage was gradually increased to $70 \mathrm{~g} /$ day $(0.9 \mathrm{~g} / \mathrm{kg} / \mathrm{d})$. AS2-1 was added to the therapeutic regime on November 3, 1989 and the dosage was gradually increased to $20 \mathrm{~g} / \mathrm{d}$ $(0.25 \mathrm{~g} / \mathrm{kg} / \mathrm{d})$. MRIs were performed every two months and tumor effects were measured by two independent neuroradiologists, one at River Oaks Imaging and Diagnostic, Houston, Texas and the other at Southwest Neuro-Imaging, Phoenix, Arizona. The patient was on BT-3 between October 30, 1989 and February 20, 1990 but subsequently continued on ANP off protocol. AS21 was permanently discontinued on March 30, 1995 while A-10 was permanently discontinued on August 25,1995 . No AEs, possibly attributable to ANP, occurred during treatment.

MRI performed on October 19, 1990 (See Figure 1 and Figure 2) demonstrated a complete response (CR). Atrophy of the brainstem with abnormal white matter signal intensity was seen, which was suggestive of hyperfractioned RT toxicity [13]. MRI performed on August 28, 1995 (See Figure 1C and Figure 2C) demonstrated persistence of the CR after five years. MRI performed on August 16, 2012 (See Figure 1D and Figure 2D) demonstrated persistence of the CR 23 years after the start of ANP.

A summary of the patient's post-treatment clinical status was provided in a letter from the patient's primary care physician dated August 15, 2012. At that time, the patient was living at home with his parents.
He spent his time in bed, in a specially adapted chair at home, or riding in a specially adapted van. The patient's father provided daily physiotherapy, which included use of a specially adapted exercise bike. The patient showed minimal movement on the left side of his body and was aphasic, communicating with a grimace or shaking of his head for "no" and with a smile or nodding of his head for "yes". He was maintained on $\mathrm{O}_{2}$ at 1.5 to 3.0 liters per minute, which kept his blood $\mathrm{O}_{2}$ saturations at 81 to $98 \%$ depending on the patient's level of activity and on the need for suction of his tracheal tube. The patient was eating a balanced diet of finely chopped food and could drink from a cup. He had not complained of headaches since treatment with ANP and was not on any regular medications. Unfortunately, the patient passed away on $1 / 21 / 2013$ due to complications related to prior RT and left-sided CVA.

\section{Conclusion}

Twenty patients, including the 26-year-old male described here, were enrolled in BT-3. Two patients had a low-grade glioma, two patients had anaplastic astrocytomas of the brainstem, and 16 patients had anaplastic astrocytomas of other areas of the brain. All patients were evaluable. The CR reported after ANP in the 26-year-old male reflects the type of outcomes seen in the other BT-3 patients. A CR was achieved in six patients $(30 \%)$, a partial response (PR) was achieved in one patient (5\%), Stable disease (SD) was achieved in nine patients $(45 \%)$, and PD was seen in four patients (20\%).

On October 4, 1991, three members of the NIH Cancer Therapy Evaluation Program (CTEP), with an invited neuropathologist and an invited neuroradiologist, visited Dr. Burzynski at the Burzynski Clinic (BC) to review seven selected brain tumors from Phase I and Phase II studies (including BT-3). Following through review, five definite or "possible" CRs were identified [16].

BT-3 led to further Phase II clinical studies, which involved a more aggressive use of ANP, including continuous infusions of higher dose A10 and AS2-1 utilizing ambulatory infusion pumps, in accordance with BT-4 [17]. In addition, there was an increased utilization of ANP in a variety of low- and high-grade brain tumors, under the Burzynski Research Institute's (BRI's) IND \# 43,742 . Multiple Phase II protocols were completed and numerous articles published [18-49].

In 2014, an unpublished review of 168 DIPG patients treated with ANP in six different Phase II protocols at BRI under IND \# 43,742, revealed that 18 patients obtained an OR (18.7\%) while 7 obtained a CR (4.2\%). Overall Survival (OS) differed between five separate patient cohorts as determined by age and prior treatment. For the 18 patients age $>21$ years who received ANP therapy for recurrent DIPG following RT \pm chemotherapy and/or other therapies, OS was $50.0 \%$ at 6 months, 


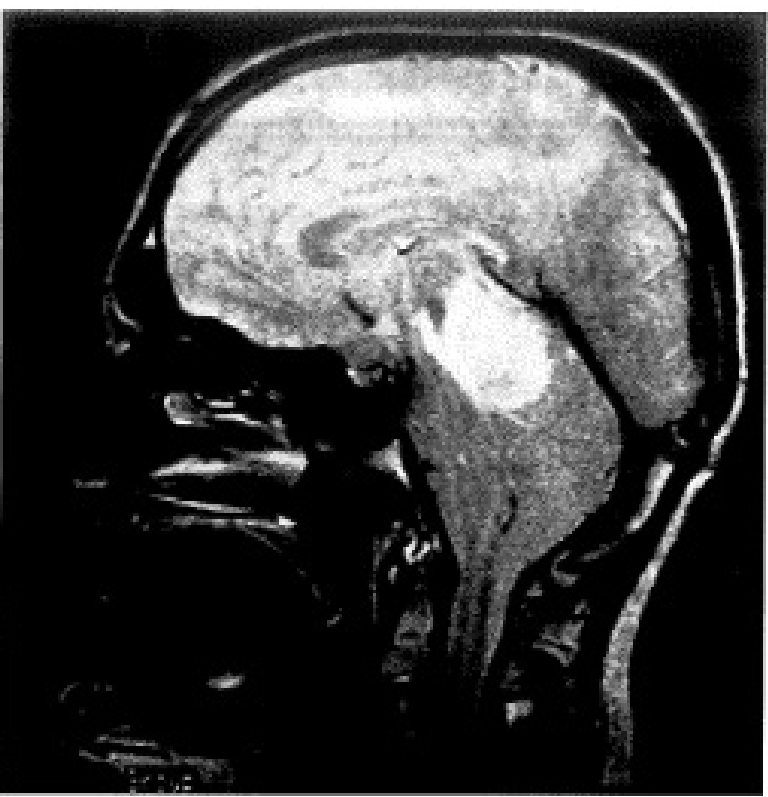

A. Sep. 23,1988

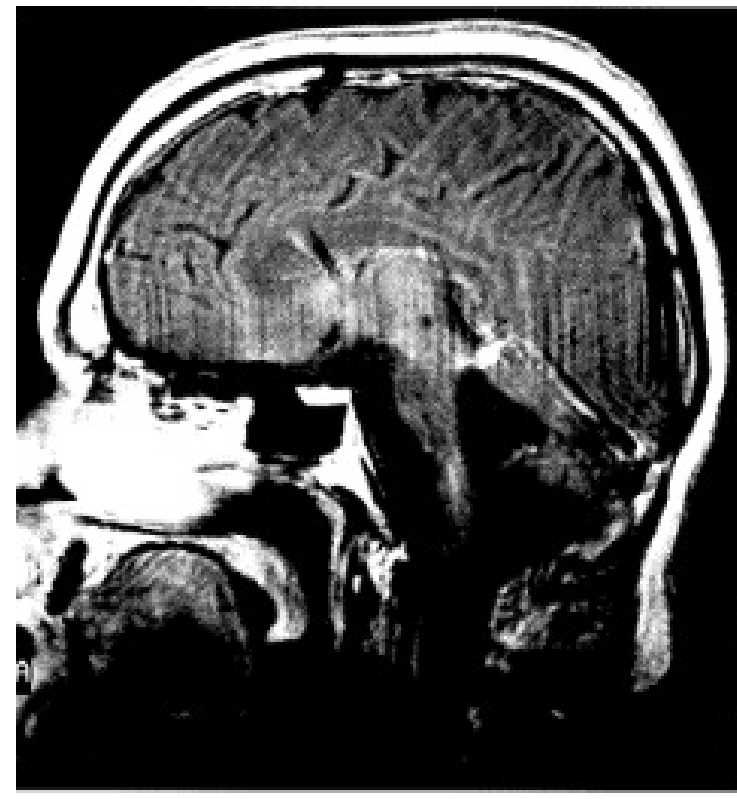

C. Aug. 28, 1995

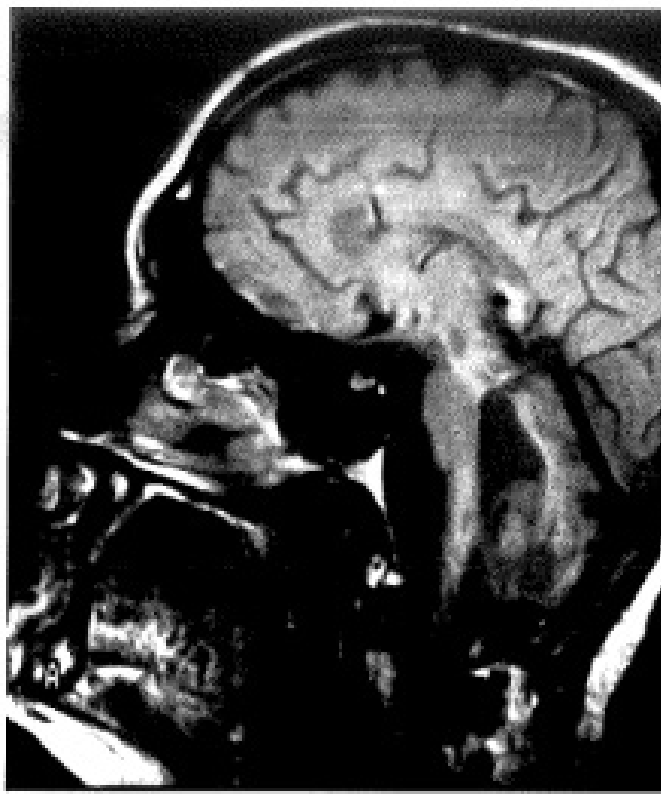

B. Oct. 19,1990

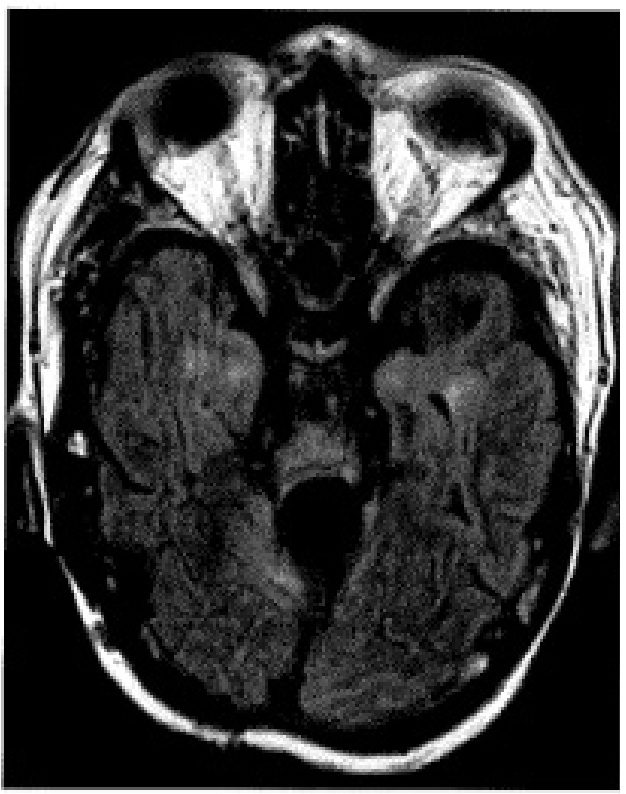

D. Aug. 16, 2012

Figure 2: Sagittal brain magnetic resonance images (MRI) T2 weighted: 2A (baseline) demonstrates a high intensity mass. $2 \mathrm{~B}$ and $2 \mathrm{C}$ are follow-up images that show a complete response (CR) of the mass to treatment. Figure 2D (FLAIR) also shows the $C R$.

$38.9 \%$ at 12 months, and $33.3 \%$ at 24 months. Based on this information, we collaborated with the FDA to develop BRI-BT-52, "A Randomized Phase 3 Study of Combination Antineoplaston Therapy [Antineoplastons A10 (Atengenal) and AS2-1 (Astugenal)] Plus Radiation Therapy vs. Radiation Therapy Only in Subjects with Newly Diagnosed Diffuse, Intrinsic Brainstem Glioma", which awaits IRB approval. Subsequent collaboration with the FDA resulted in "A Phase 2 Study of Atengenal (A-10) and Astugenal (AS2-1) in Diffuse, Intrinsic, Brainstem Glioma (DIPG)", which has received IRB approval and will be opened for patient accrual in the near future.
ANP is an attractive therapeutic option for those patients with high-grade astrocytoma (a glial tumor with astrocytic differentiation), including DIPG, who are ineligible for or refuse RT, and/or demonstrate PD following RT or chemotherapy. We have presented here the case of a 26-year-old male who obtained long term survival ( 23 years) following ANP for DIPG. This and similar cases led to further Phase II clinical studies, which involved a more aggressive use of ANP, including continuous infusion of higher doses of A10 and AS2-1 utilizing ambulatory infusion pumps, and an increased utilization of ANP in a variety of low- and high-grade brain tumors under the Burzynski Research Institute's (BRI's) IND \# 43,742. 


\section{Acknowledgment}

The authors express their appreciation to Carolyn Powers for preparation of the manuscript and to Ramiro Rivera, Mohamed Khan, Jennifer Pineda and Adam Golunski for their involvement.

\section{References}

1. Hoffman LM, Veldhuijzen van Zanten SEM, Colditz $N$, Baugh J, Chaney B, et al. (2018) Clinical, radiologic, pathologic, and molecular characteristics of long-term survivors of diffuse intrinsic pontine glioma (DIPG): A collaborative report from the international and European society for pediatric oncology DIPG registries. J Clin Oncol 36: 1963-1972.

2. Jansen $M H$, Veldhuijzen van Zanten SE, Sanchez Aliaga E, Heymans MW, Warmuth-Metz M, et al. (2015) Survival prediction model of children with diffuse intrinsic pontine glioma based on clinical and radiological criteria. Neuro Oncol 17: 160-166.

3. Langmoen IA, Lundar T, Storm-Mathisen I, Lie SO, Hovind KH (1991) Management of pediatric pontine gliomas. Childs Nerv Syst 7: 13-15.

4. Smith MA, Freidlin B, Ries LA, Simon R (1998) Trends in reported incidence of primary malignant brain tumors in children in the United States. J Natl Cancer Inst 90: 12691277.

5. Ostrom OT, Patil N, Cioffi G, Waite K, Kruchko C, et al. (2020) CBTRUS statistical report: Primary brain and other central nervous system tumors diagnosed in the United States in 2013-2017. Neuro Oncol 22: iv1-iv96.

6. RoujeauT, Machado G, Garnett MR, Miquel C, Puget S, et al. (2007) Stereotactic biopsy of diffuse pontine lesions in children. J Neurosurg 107: 1-4.

7. Cage TA, Samagh SP, Mueller S, Nicolaides T, HaasKogan D, et al. (2013) Feasibility, safety, and indications for surgical biopsy of intrinsic brainstem tumors in children. Childs Nerv Syst 29: 1313-1319.

8. Louis DN, Perry A, Reifenberger G, von Deimling A, Figarella-Branger D, et al. (2016) The 2016 world health organization classification of tumors of the central nervous system: A summary. Acta Neuropathol 131: 803-820.

9. Castel D, Philippe C, Calmon R, Le Dret L, Truffaux N, et al. (2015) Histone H3F3A and HIST1H3B K27M mutations define two subgroups of diffuse intrinsic pontine gliomas with different prognosis and phenotypes. Acta Neuropathol 130: 815-827.

10. Karremann M, Gielen GH, Hoffmann M, Wiese M, Colditz $\mathrm{N}$, et al. (2018) Diffuse high-grade gliomas with H3 K27M mutations carry a dismal prognosis independent of tumor location. Neuro Oncol 20: 123-131.

11. von Bueren $A O$, Karremann $M$, Gielen $G H$, Benesch $M$, Fouladi $M$, et al. (2018) A suggestion to introduce the diagnosis of "diffuse midline glioma of the pons, H3 K27 wildtype (WHO grade IV)". Acta Neuropathol 136: 171-173.

12. Burzynski SR, Janicki T, Burzynski GS, Beenken S (2021) Long-term survival (27.7 years) following IV antineoplaston therapy (ANP) in a 36-year-old-female with a progressive diffuse intrinsic pontine glioma (DIPG). Int J Radiol Imaging Technol 7: 073.

13. Kesari S, Kim RS, Markos V, Drappatz J, Wen PY, et al. (2008) Prognostic factors in adult brainstem gliomas:
A multicenter retrospective analysis of 101 cases. J Neurooncol 88: 175-183.

14. Burzynski SR, Patil S (2014) The effect of Antineoplaston A10 and AS2-1 and metabolites of sodium phenylbutyrate on gene expression in glioblastoma multiforme. J Cancer Ther 5: 929-945.

15. Burzynski SR, Janicki T, Burzynski G (2015) Comprehensive genomic profiling of recurrent classic glioblastoma in a patient surviving eleven years following antineoplaston therapy. Cancer Clin Oncol 4: 41-52.

16. Hawkins MG, Friedman MA (1992) National cancer institute's evaluation of unconventional cancer treatments. J Natl Cancer Inst 84: 1699-1702.

17. Burzynski SR, Kubove E, Szymkowski B (1993) Phase II clinical trials of antineoplaston A10 and AS2-1 infusion in high grade glioma. Presented at the 18th international Congress of Chemotherapy, Sweden.

18. Burzynski SR, Conde AB, Peters A, Saling B, Ellithorpe R, et al. (1999) A Retrospective Study of Antineoplastons A10 and AS2-1 in Primary Brain Tumors. Clin Drug Investig 18: 1-10.

19. Burzynski SR, Weaver RA, Bestak M, Lewy RI, Janicki TJ, et al. (2003) Phase II study of Antineoplastons A10 and AS2-1 (ANP) in children with recurrent and progressive multicentric glioma. A preliminary report. Neuro-Oncology 5: 358.

20. Burzynski SR, Lewy RI, Weaver R, Janicki T, Jurida G, et al. (2004) Long-term survival and complete response of a patient with recurrent diffuse intrinsic brain stem glioblastoma multiforme. Integ Cancer Ther 3: 257-261.

21. Burzynski SR, Weaver R, Lewy R, Janicki T, Jurida G, et al. (2004) Phase II study of antineoplaston A10 and AS2-1 in children with recurrent and progressive multicentric glioma. A Preliminary Report. Drugs R D 5: 315-326.

22. Burzynski SR, Weaver R, Bestak M, Janicki T, Jurida G, et al. (2004) Phase II studies of antineoplastons A10 and AS21 (ANP) in children with atypical teratoid/rhabdoid tumors (AT/RT) of the central nervous system. A preliminary report. Neuro Oncology 6: 427.

23. Burzynski SR, Weaver R, Bestak M, Janicki T, Szymkowski $B$, et al. (2004) Treatment of primitive neuroectodermal tumors (PNET) with antineoplastons A10 and AS2-1 (ANP). Preliminary results of phase II studies. Neuro Oncology 6 : 428.

24. Burzynski SR, Weaver RA, Janicki T, Szymkowski B, Jurida $G$, et al. (2005) Longterm survival of high-risk pediatric patients with primitive neuroectodermal tumors treated with antineoplastons A10 and AS2-1. Integ Cancer Ther 4: 168177.

25. Burzynski SR (2006) Targeted therapy for brain tumors. In: Yang AV, Brain cancer therapy and surgical interventions. Nova Science Publishers, New York.

26. Burzynski SR, Janicki, TJ, Weaver RA, Burzynski B (2006) Targeted therapy with antineoplastons A10 and AS2-1 of high grade, recurrent, and progressive brainstem glioma. Integ Cancer Ther 5: 40-47.

27. Burzynski SR (2006) Treatments for astrocytic tumors in children: Current and emerging strategies. Ped Drugs 8: 167-178.

28. Burzynski SR (2007) Recent clinical trials in diffuse intrinsic brainstem glioma. Cancer Ther 5: 379-390.

29. Burzynski S, Janicki T, Burzynski G, Marszalek A (2014) 
Long-term survival ( $>13$ years) in a child with recurrent diffuse pontine gliosarcoma: A case report. J Ped Hematol Oncol 36: e433-e439.

30. Burzynski SR, Janicki TJ, Burzynski GS, Marszalek A (2014) A phase II study of antineoplastons A10 and AS21 in children with high-grade glioma. Final report (Protocol BT-06) and review of recent trials. J Cancer Ther 5: 565577.

31. Burzynski SR, Janicki TJ, Burzynski GS (2014) A phase II study of antineoplastons A10 and AS2-1 in adult patients with recurrent glioblastoma multiforme: Final report (Protocol BT-21). J Cancer Ther 5: 946-956.

32. Burzynski SR, Burzynski GS, Janicki TJ (2014) Recurrent glioblastoma multiforme-A strategy for long-term survival. J Cancer Ther 5: 957-976.

33. Burzynski SR, Janicki TJ, Burzynski, GS, Marszalek A, Brookman S (2014) A phase II study of antineoplastons A10 and AS2-1 in children with recurrent, refractory or progressive primary brain tumors-final report (Protocol BT22). J Cancer Ther 5: 977-988.

34. Burzynski SR, Janicki TJ, Burzynski GS, Brookman S (2014) Preliminary findings on the use of targeted therapy with pazopanib and other agents in combination with sodium phenylbutyrate in the treatment of glioblastoma multiforme. J Cancer Ther 5: 1423-1437.

35. Burzynski GS, Janicki TJ, Marszalek A (2014) Long-term survival ( $>20$ years) of a child with brainstem glioma treated with antineoplastons A10 and AS2-1: A case report. Neuro Oncology 11: 16.

36. Burzynski SR, Janicki TJ, Burzynski GS, Marszalek A (2014) The response and survival of children with recurrent diffuse intrinsic pontine glioma based on phase II study of antineoplastons A10 and AS2-1 in patients with brainstem glioma. Childs Nerv Syst 30: 2051-2061.

37. Burzynski SR, Burzynski G, Janicki J, Marszalek A (2015) Complete response and Long-term survival ( $>20$ years) of a child with tectal glioma: A case report. Pediatr Neurosurg 50: 99-103.

38. Burzynski SR, Janicki TJ, Burzynski G (2015) A phase II study of antineoplastons A10 and AS2-1 injections in adult patients with recurrent anaplastic astrocytoma-final report (Protocol BT-15). Cancer Clin Oncol 442: 13-23.

39. Burzynski SR, Janicki TJ, Burzynski GS, Marszalek A (2015) A Phase II Study of Antineoplastons A10 and AS2-1 in adult patients with Newly-Diagnosed Anaplastic Astrocytoma Final Report (Protocol BT-08). Cancer Clin Oncol 4: 28-38.
40. Burzynski SR, Burzynski GS, Marszalek A, Janicki J, Martinez-Canca J (2015) Long-term survival (over 20 years), complete response and normal childhood development in medulloblastoma treated with antineoplastons A10 and AS2-1. J Neurol Stroke 2: 00054.

41. Burzynski SR, Burzynski GS, Marszalek A, Janicki TJ, Martinez-Canca JF (2015) Long-term survival over 21 years and pathologically confirmed complete response in pediatric anaplastic astrocytoma: A case report. J Neurol Stroke 2: 00072.

42. Burzynski SR, Burzynski GS, Brookman S (2015) A case of sustained objective response of recurrent/progressive diffuse intrinsic pontine glioma with phenylbutyrate and targeted agents. J Cancer Ther 6: 40-44.

43. Burzynski SR, Janicki T, Burzynski G, Marszalek A (2015) A phase II study of antineoplastons A10 and AS2-1 in patients with brainstem gliomas. The report on non-diffuse intrinsic pontine glioma (Protocol BT-11). J Cancer Ther 6: 334-344.

44. Burzynski SR, Janicki T, Burzynski G (2015) A phase II study of antineoplastons $A 10$ and $A S 2-1$ in adult patients with primary brain tumors. Final report (Protocol BT-09). J Cancer Ther 6: 1063-1074.

45. Burzynski SR, Janicki TJ, Burzynski GS (2016) Primary CNS tumors and leptomeningeal, disseminated and/or multicentric disease in children treated in phase ii studies with antineoplastons A10 and AS2-1. Cancer Clin Oncol 5: 38-48.

46. Burzynski SR, Janicki TJ, Burzynski GS (2016) A phase II study of antineoplastons A10 and AS2-1 in children with low-grade astrocytomas-final report (Protocol BT-13). J Cancer Ther 7: 837-850.

47. Burzynski SR, Janicki TJ, Burzynski GS (2017) Antineoplastons A10 and AS2-1 in the treatment of children with optic pathway glioma: Final report for protocol BT-23. Cancer Clin Oncol 6: 25-35.

48. Burzynski SR, Janicki TJ, Burzynski GS, Marszalek A (2017) A phase II study of antineoplastons A10 and AS2-1 in children with brain tumors. Final report (Protocol BT-10). J Cancer Ther 8: 173-187.

49. Burzynski SR, Janicki T, Beenken S (2019) Treatment of recurrent glioblastoma multiforme (rGBM) with antineoplaston AS2-1 in combination with targeted therapy. Cancer Clin Oncol 8: 1. 